\title{
Pompes à métaux liquides
}

\section{Pumps for liquid metals}

\author{
PAR C. HERMANT
}

INGÉNIEUR AU SERVICE DES LabonatoIRES ET ESSAIS HYDRAULIQUES DE L'V́LECTRIGITY DE FRANCE

\begin{abstract}
Le transfert de chaleur par métaux liquides a été envisagé, depuis quelques années, notamment en vue de produire de l'énergie d'origine nucléaire. Les pompes nécessaires à la mise en circulation da métal liquide - sodium, bismuth, etc... - dans un circuit d'échange thermique, doivent présenter des caractéristiques singulières et, en particulier, une étanchéité totale et une sécurité absolue de fonctionnement.

Des pompes mécaniques, $d u$ type centrifuge classique, mais comportant un dispositif original d'étanchéité, ont été proposées; les différents types connus sont décrits dans la première partie de l'exposé.

Par suite de leur cunductivité électrique relativement élevée, les métaux liquides peuvent être mis en circulation par action électromagnétique directe et sans l'intermédiaire d'aucune partie tournante. La deuxième partie de l'exposé comprend la deseription des types principaux de pompes étectromagnétiques, et la présentation de quelques résultats d'étude concernant les pompes électromagnétiques ì induction.

L'article se termine par une comparaison sommaire entre ces différents dispositifs de pompage.
\end{abstract}

On sait que des métaux liquides sont expérimentés, depuis quelques années, comme moyen de transfert thermique, notamment en vue de l'utilisation de l'énergie nucléaire $\left(^{*}\right)$. Si leurs caractéristiques physiques, thermiques et nucléaires leur confèrent un certain intérêt, d'autres propriétés (réactivité chimique, radioactivité induite, toxicité...) compliquent le problème

(*) On désigne sous le nom de « métaux liquides 》 ceux dont la température de fusion est relativement hasse (approximativement inférieure à $350^{\circ} \mathrm{C}$ ). Le sodium

\begin{abstract}
Heat transfer using liquid metals has been considered for several years mainly in connection with nuclear power. The pumps required for circulating the liquid metal (sodium, bismuth, etc.) in a heat exchanger circuit must have special properties, of which the main ones are a total absence of leaks and complete reliability. Conventional centrifugal mechanical pumps which incorporate an original type of seal have been proposed; the various pumps are described in the first part of the paper.
\end{abstract}

On account of their relatively high electrical conductivity, liquid metals can be circulated by electromagnetism alone directly and without using any rotating components. The second section describes the main types of electromagnetic pumps and gives the results of research on electromagnetic induction pumps. The article ends with a brief comparison of the various pumps described.

de leur mise en ouvre. Elles appellent en particulier la mise au point de nouveaux moyens de pompage destinés à mettre le métal liquide en circulation dans le circuit d'échange thermique $(1,2,3)$.

Avant de présenter les différentes solutions envisagées, le problème posé peut être précisé en énonçant d'une manière générale les diverses qualités que l'on demande à une pompe à métaux liquides.

et le bismuth sont à citer particulièrement, ainsi que les alliages sodium-potassium (NaK) et plomb-bismuth. 


\section{I. - QUALITÉS NECESSAIRES}

Bien que d'autres applications soient envisagées, on considérera principalement le transfert thermique à partir d'un réacteur nucléaire, problème qui est à l'origine du développement de l'utilisation des métaux liquides $(4,5)$.

\section{1. - Etanchéité}

Une exigence que l'on place habituellement en lète est celle de l'étanchéité absolue du système de pompage. Les raisons en sont bien connues, et il paraît inutile d'insister sur les conséquences funestes d'une fuite de métal liquide - tel le sodium - porté à haute température, radioactif et réagissant violemment avec l'eau et l'air.

Une fuite, mème minime, permettrait, de plus, une rentrée d'oxygène dans le circuit d'échange thermique, ce qui doit être rigoureusement proscrit. En effet, la présence d'oxydes dans le circuit de métal liquide déclencherait de riolentes corrosions. On admet ainsi que la teneur maximale en oxygène est de l'ordre de $0,001 \%$ seulement.

\section{2. - Sécurité}

L'utilisation de métaux liquides pour le refroidissement d'un réacteur nucléaire nécessite un moyen de pompage présentant toute garantie au point de vue sécurité de fonctionnement. On cherche à réduire l'éventualité d'une défaillance qui pourrait provenir d'une résistance insuffisante des matériaux utilisćs, à la corrosion et à la radioactivité, ou encore d'une cause purement mécanique.

\section{3. - Entretien}

Par suite des sujétions qu'il présente dans le cas d'une installation de transfert thermique fonctionnant généralement d'une manière continue, l'entretien d'une pompe à métaux liquides devra être aussi réduit que possible. Néanmoins et d'une manière générale, on admet de plus en plus l'entretien d'une installation atomique, à condition de prévoir des moyens d'intervenir sûrs, économiques et rapides. S'il est nécessaire, l'entretien des pompes devra done être facilití au maximum, par une étude particulière de l'accessibilité et du démontage des divers organes.

\section{4. - Caractéristiques}

Le service qu'on demande aux pompes d'assurer dans de bonnes conditions d'étanchéité, de sécurité, d'entrelien, est par ailleurs défini par certaines caractéristiques.

Citons, par exemple, un projet amćricain de réacteur de puissance refroidi par sodium liquide (Sodium graphite reactor). La puissance thermique préve est de $250.000 \mathrm{~kW}$, ce qui conduit à une puissance électrique netle de $75.000 \mathrm{~kW}$; les pompes du circuit de refroidissement du réacteur doivent débiter $950 \mathrm{l} / \mathrm{s}$ $(3.000 \mathrm{t} / \mathrm{h})$ de sodium dont la température varie de $260^{\circ}$ à l'admission, à $500^{\circ}$ à la sortie du réacteur.

Nous allons maintenant présenter les principaux types de jompes à métaux liquides et les comparer au point de vue de leurs caractéristiques d'utilisation : métanx liquides ulilisables, température, débit et pression réalisables (6). On considérera également leurs performances : rendement, puissance massique et coût relatif.

\section{II. - POMPES MÉCANIQUES}

Les pompes mécaniques pour métaux liquides sont du type centrifuge qui parait bien adapté au problème, notamment en ce qui concerne les caractéristiques de pression et débit. Le problème essentiel est de réaliser une liaison absolument étanche entre le moteur d'entrainement et la roue centrifuge. Les dispositifs usuels du genre presse-étoupe - ne conviennent yas pour des pompes à métaux liquides : l'ćtanchéité obtenue est insuffisante, et, de plus, les corps utilisés pour réaliser des joints at frottement (amiante, caoutchouc, téflon, grajphite...) sont détériorés par les métaux liquides à haute température. Seules certaines tresses métalliques comprimées (nickel, cuivre) résistent, mais s'usent rapidement. Des solutions originales ont été étudiées, qui peuvent être classées en trois types principaux $(2,3)$. 


\section{A. - Pompes à rotor immergé}

La partic lournante du groupe moto-pompe est entièrement immergée; le stator du moteur d'entraînement - de type asynchrone - est séparé du mélal liquide par une membrane

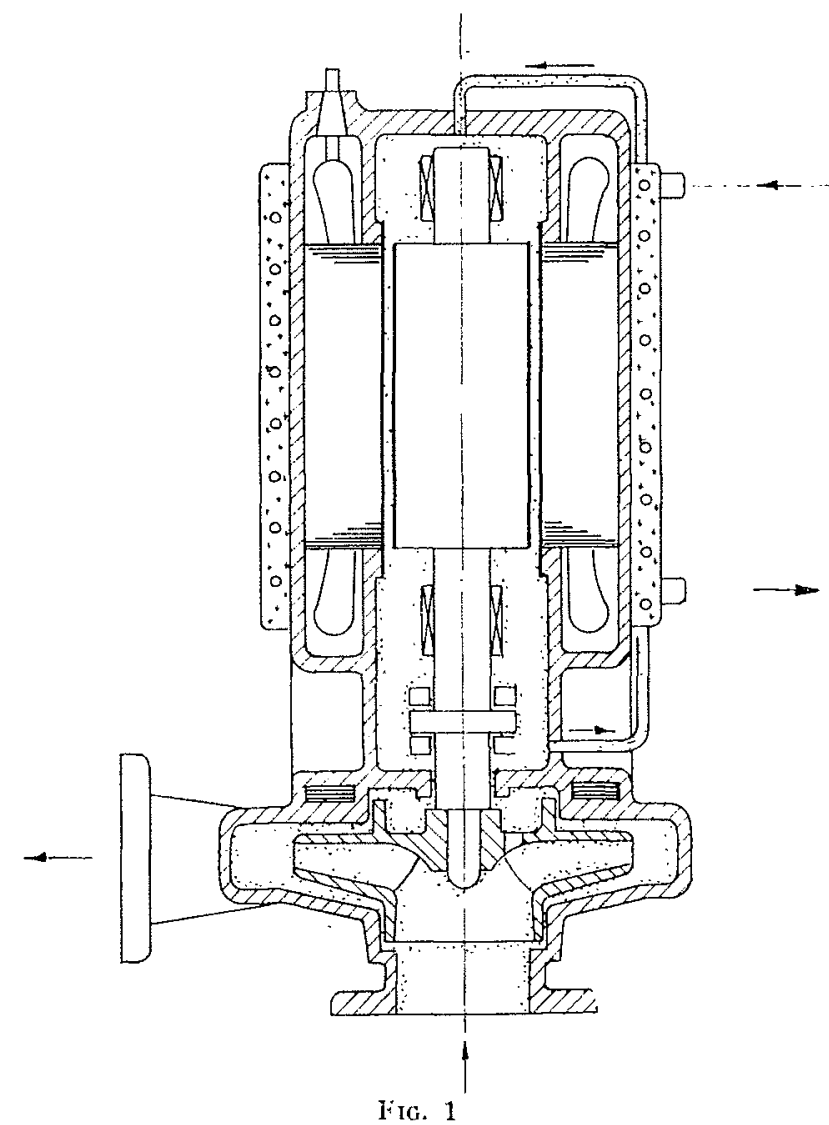

Pompe à rotor immergé.

(Figure extraite de Liquid Metals llandbook.)

mince placće dans l'entrefer (fig. 1). Le problime essentiel posé par ce type de pompe est celui de la lubrification des paliers el des butées par le métal liquide lui-même, peu approprié à cet usage. Dans les machines importantes, l'axe do la pompe est centrée à l'intérieur de paliers hydrodynamiques alimentés en métal liquide par lc refoulement de la pompe. Pour assurer une honne lubrification des paliers et éviter de surchauffer les enroulements, on réalise une circulation auxiliaire du métal liquide qui baigne le rotor, à travers un échangeur, de manière à abaisser la températurc à $150^{\circ} \mathrm{C}$ environ. La durée de vie des paliers de la pompe est limitéc par des phénomènes de corrosion, dont l'importance est essentiellement fonction de la teneur en oxyde du métal liquide. Anssi à l'échangeur dont il vient d'être question on associe généralement « un piège froid», qui limite la teneur en oxyde par solidification et filtration.

Le rendement d'un tel groupe moto-pompe est plus faible que celui d'un groupe classique, par suite de la présence du diaphragme et d'un film de métal liquide dans l'entrefer du moteur. Il s'ensuit des pertes par frottement et par courants de Foucault qui limitent le rendement à une valeur de l'ordre de 50 à $60 \%$. Un autre inconvénient de ce type de machine consiste en difficultés d'entretien de la partie tournante et des paliers; les joints entre les différentes parlies sont généralement remplacés par des soudures d'étanchéilé qui compliquent les démontages éventuels.

Ce type de machine est réalisé couramment en petites dimensions dont les caractéristiques sont de l'ordre de quelques litres/seconde, sous une hauteur manométrique de 20 à 30 mètres. La Société Westinghouse a annoncé récemment la mise au point d'une pompe de ce type, plus importante, dont le débit s'élève à 300 litres/seconde, la température maximum du métal liquide alteignant $850^{\circ} \mathrm{C}$.

\section{B. - Pompes à couverture gazeuse}

A priori plus simple, le principe consiste à reporter le problème d'étanchéité sur un gaz

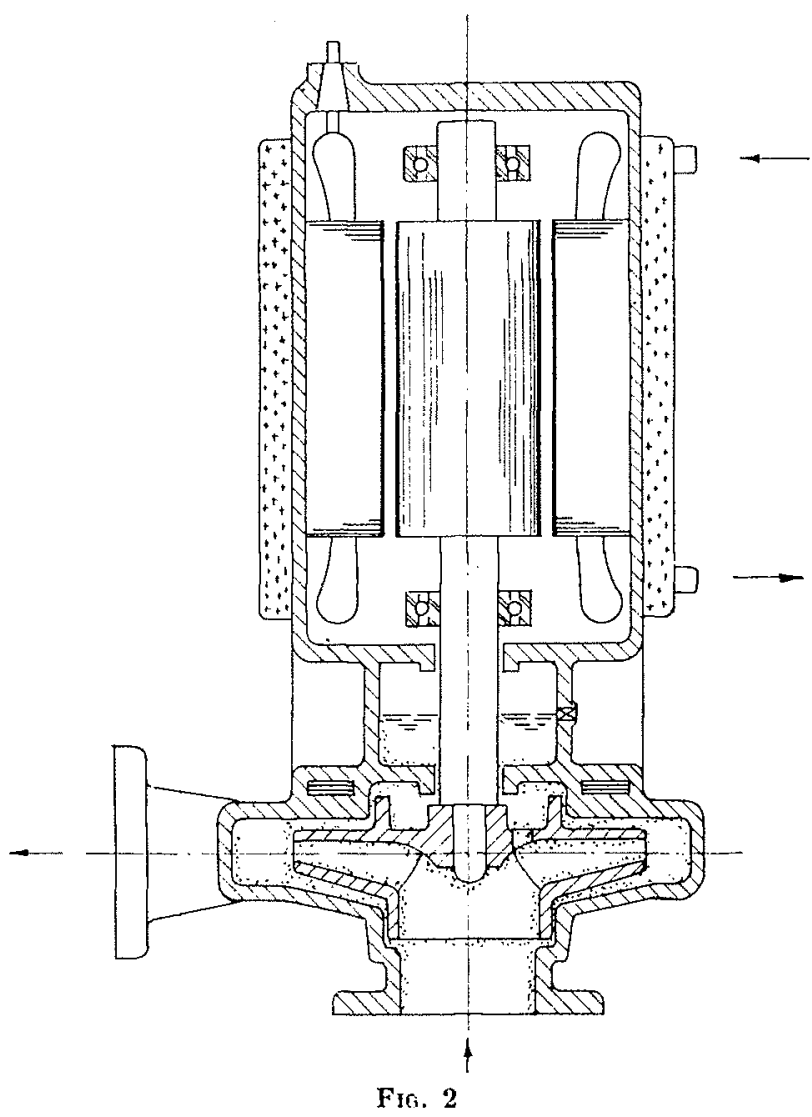

Pompe à couverture gareuse.

(Figure extraite de Liquid Metals Mandbook.)

inerte recouvrant le métal liquide à la partie supérieure de la pompe (fig. 2). L'arbre de la 
pompe centrifuge est vertical et pénètre dans le carter à travers un joint. Celui-ci peut être du genre presse-étoupe, si l'on admet une certaine fuite de gaz inerte; sa lubrification est difficile par suite du risque de contamination du métal liquide. Si le métal liquide est radioactif, on ne peut admettre la moindre fuite de gaz, et il paraît préférable de placer également le moteur d'entraînement dans un carter étanche rempli de gaz inerte, à la même pression que le carter de la pompe. Un joint labyrinthe est cependant nécessaire entre le carter du moteur et celui de la pompe, pour limiter la diffusion de vapeur de sodium et éviter sa condensation sur les enroulements et les palier's du moteur d'entrainement.

Les problèmes posés par ce type de pompe peuvent s'énoncer ainsi : graissage du joint, diffusion $\mathbf{d u}$ «métal liquide 》 dans le carter moteur à haute température, risque d'entrainement de gaz, nécessité de contrôler le niveau du liquide qui peut varier suivant le régime de fonctionnement. Par contre, cette pompe présente l'avantage d'être de fabrication simple utilisant des éléments existants; son rendement est comparable à celui des pompes classiques.

\section{C. - Pompes à joint de sodium solidifié}

L'étanchëité entre l'arbre d'entraînemenl de la pompe centrifuge et le carter est assurée en solidifiant localement le liquide. Cette solution expérimentée pour le pompage du sodium, consiste à remplacer le presse-étoupe habituel par un manchon refroidi par une circulation auxiliaire. (On utilise par exemple du toluène qui ne réagit pas avec le sodium.) Le sodium qui se solidifie dans cette région sous forme d'un mince anneau, réalise un joint d'une étanchéité acceptable. Les tolérances mécaniques d'usinage de l'arbre et du manchon sont assez strictes: si le jeu est trop faible, on risque blocage; s'il est trop élevé, il peut y avoir extrusion progressive de sodium. La puissance calorifique ainsi produite par frottement peut être de l'ordre du kilowatt, dans le cas d'une pompe de puissance importante $(30 \mathrm{~kW})$; si la température du métal liquide est élevée, $500^{\circ}$ par exemple, il faut alors évacuer un flux calorifique de plusieurs. kilowatts, pour maintenir le sodium à l'état liquide. Au démarrage, le couple est comparable à celui d'une pompe classicque dont les garnitures seraient serrées. Pour éviter le contact du sodium avec l'air et pour limiter la différence de pression de part et d'autre du joint, une enceinte remplie de gaz inerte sous pression est aménagée derrière le joint. Ce type de pompe semble présenter de nombreux avantages : rendement élevé, entretien facile, fabrication relativement simple et bas prix. Par ailleurs, la sécurité paraît suffisante : l'arrêt du refroidissement n'entraine pas un incident immédiat et quelques minutes restent disponibles pour prendre des mesures d'urgence.

Par contre, ne sont utilisables que les métaux liquides à température de fusion relativement élevée et qui ne comportent pas de matériaux abrasifs ou d'oxydes se déposant dans les zones froides. Dans l'ensemble, ce type de machine parait assez prometteur, mais semble nicessiter encole une certaine expérience.

\section{D. - Autres solutions}

A part les trois types qui viennent d'être déerits, quelques antres solutions ont été envisagées, dont l'avenir paraît plus limité.

On peut penser transmetlre un effort au métal liquide par intermédiaire d'une partie déformable, suivant le principe adopté pour la fabrication de ccrtaines vannes à étanchéité totale (vannes à soufflets ou à membrane). Si cetle solution est satisfaisante dans le cas d'une vanne à fonctionnement intermittent, elle ne paraît pas prisenter une sécurité salisfaisante dans le cas du pompage.

De simples pompes alternatives sont utilisces dans certaines installations chimiques pour le transfert de métatux liquides à température modérée $\left(200^{\circ}\right)$; les fuites de métal liquide entre piston et eylindre sont collectées el renvoyées a un réservoir; un gaz inerte remplit la partic arrière du cylindre qui est approximativement ćtanche, grâce à un joint à garnitures dans lequel coulisse la tige du piston. Ce système constitue dans certains cas un moyen simple d'obtenir un débit connu sous une pression qui peul être ćlevée, mais à une température limitée.

\section{III. - POMPES ELECTROMAGNETIQUES}

\section{A. - Généralités}

Une autre solution au problème du pompage des métaux liquides --solution assez séduisante $a$ priori - consiste à mettre le liquide conducteur directement en mouvement par action élec- tromagnétique, sans l'inlermédiaire d'aucune partie tournante. La conductivité électrique élevée de certains mélaux liquides, intéressants à d'autres points de vue, rend pratiquement possible un tel dispositif. Cette solution avait séduit M. Remevieras, chel du Service des Eludes 
Hydrauliques de l'Electricité de France, quì dès 1946 orientait une partie des recherches vers la mise au point d'appareils de ce genre (10).

L'idée de transmettre un effort à un liquide conducteur par l'action d'un champ magnétique sur un courant électrique est assez ancienne. La pression hydrostatique, engendrée par effet de pincement, fut utilisce par NoRThrup vers 1900 pour mesurer des courants alternatifs intenses (7). Par la suite, dautres applications furent envisagées $(8,9)$, mais c'est toùt récemment que l'intérêt du pompage électromagnétique s'est accru, à la suite des problèmes de transfert thermique posés par l'utilisation de l'énergie nucléaire.

i.es pompes électromagnétiques sont analogues aux moteur's électriques; leur fonctionnement est également régi par la loi de Laplace qui exprime la force exercée sur un conducteur traversé par un courant et placé dans un champ magnétique.

Comme dans le cas des moteurs électriques, il existe divers types de pompes électromagnétiques $(11,12)$. Elles peuvent être du type « à conduction. 》(type Faraday) où un courant électrique très intense fourni par une machine spéciale (redresseur ou génératrice homopolaire) traverse le métal liquide soumis par ailleurs à un champ magnétique. D'autres sont du type «à induction » et analogues à un moteur asynchrone; les courants électriques sont alors induits dans le liquide par les variations d'un champ magnétique produit, par exemple, par un enroulement polyphasé analogue à un "stator » de moteur à induction $(13,14)$.

\section{1) Equations gÉNérales DES POMPES ÉLECROMAGNÉTIQUES}

Considérons un élément parallélépipédique $d x d y d z$ de métal liquide situé dans la partie active de la pompe (fig. 3). Il est soumis à une

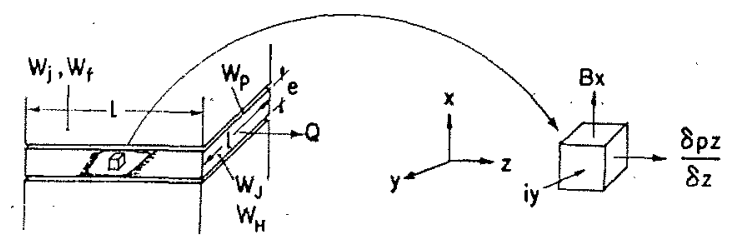

FIa. 3

Eléments du calcul des pompes électromagnétiques.

induction magnétique et à une densité de courant, dont on ne considère que les composantes $\mathrm{B}_{x}$ et $i_{y}$. L'équation de la force s'exerçant sur cet élément nous donne le gradient de pression dans la direction $z$ :

$$
\frac{\partial p_{z}}{\partial_{z}}=\mathrm{B}_{w} i_{i \prime}
$$

En intégrant ces efforts élémentaires sur la longueur totale $\mathrm{L}$, on oblient la différence de pression $\mathrm{P}$ produite par la pompe :

$$
\mathrm{P}=\int_{0}^{\mathrm{L}} \mathrm{B}_{x} \mathrm{i}_{y} d z
$$

Si la pression est uniforme dans chaque scetion droite du tube, la puissance hydraulique transmise au métal liquide est égale au produit du débit traversant une section droite par cette différence de pression $P$.

Une partie de la puissance électromagnétique fournie au fluide est perdue sous forme de pertes par courants de Foucault dans le métal liquide, pertes dont l'expression élémentaire est :

$$
d \mathrm{~W}=\mathrm{\vartheta}^{i^{2}} d x d y d z
$$

Les autres pertes électriques comprennent les pertes Joule dans l'enroulement inducteur, les pertes par courants de Foucault dans le conduit généralement métallique, et les pertes par courants de Foucault et hystérésis dans la partie magnétique.

En outre, une partie de la puissance hydraulique transmise au métal liquide est perdue sous forme de pertes de charge à l'intérieur même de la pompe.

\section{2) Pirtes hydraulioues daNS les pompes ÉleC- TROMAGNÉTIQUES :}

La méthode usuelle de calcul peut conduire à une erreur notable, si on ne tient pas compte de la modification du régime d'écoulement résultant de l'action du champ magnétique sur le liquide conducteur.

Des mouvements turbulents agitent le liquide

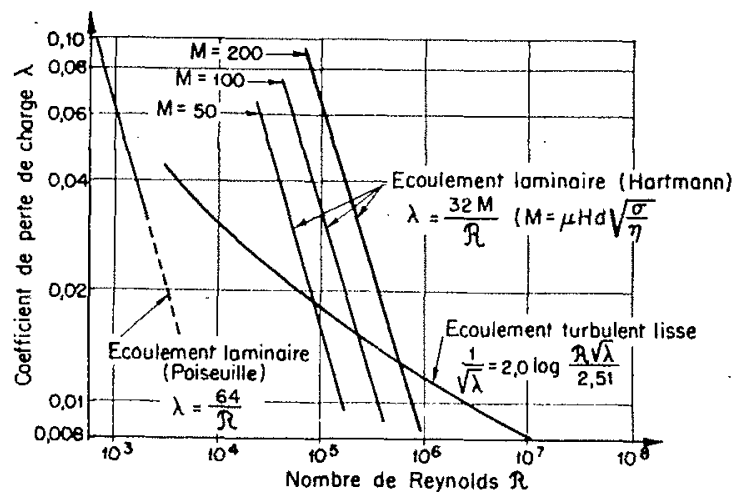

FIG. 4

Régimes d'écoulement d'un liquide conducteur soumis a un champ magnétique.

aux nombres de Reynolds élevés qui caractérisent l'écoulement dans la pompe. Ils produisent par induction des courants électriques qui, par réaction avec le champ magnétique, engendrent des efforts résistants. La turbulence est retar- 
dée, le régime d'écoulement devient laminaire avec, en général, une augmentation du coefficient de pertes de charge.

Divers auteurs $(15,16)$. ont étudié les effets d'un champ magnétique sur les liquides conducteurs en mouvement, et la variation corrélative du coefficient de pertes de charge. Dans le cas du régime « turbulent lisse » (fig. 4), on passerait progressivement de la formule de Karman :

$$
\frac{1}{\sqrt{\lambda}}=2 \frac{\log R \sqrt{\lambda}}{2,51}
$$

à la formule théorique suivante due à Hartmann :

$$
\lambda=\frac{32 \mathrm{M}}{\mathrm{R}}
$$

On désigne par $\lambda$ le coefficient de la formule universelle de pertes de charge, $R$ le nombre de Reynolds et $M$ un nombre sans dimensions caractéristiques de l'effet électromagnétique.

$$
\mathrm{M}=\mu \mathrm{H} d \sqrt{\frac{\sigma}{\eta}}
$$

$H$ désigne le champ magnétique,

$d$ désigne le diamètre hydraulique de la vcine liquide,

u, $\sigma, \eta$ désignent respectivement la perméabilité, la conductivité, la viscosité absolue du fluide.

Ces considérations ont été convenablement vérifiées dans le cas du mercure et permettent, en l'absence d'expériences complémentaires, d'estimer plus correctement les pertes de charge à l'intérieur d'une pompe électromagnétique.

\section{B. - Pompes à conduction à courant continu}

Comme le montre la figure 5 , le fonctionnement de la pompe à conduction résulte de l'application de la loi d'électromagnétisme connue sous le nom de règle des trois doigts. Si on applique à un conduit contenant un liquide conduc-

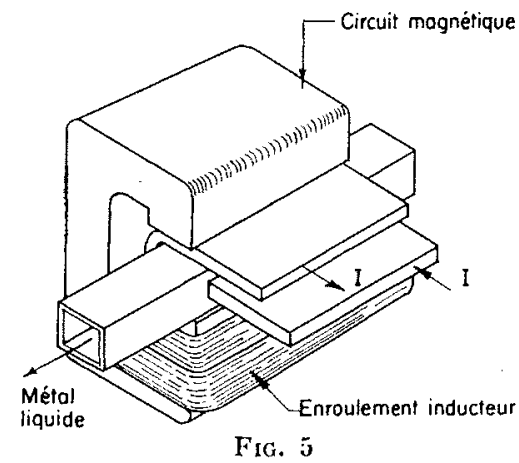

Pompe électromagnétique à conduction. Type Faraday teur un champ magnétique transversal, et si on fait passer dans le même liquide un courant électrique perpendiculaire à la fois au conduit et au champ magnétique, le conducteur liquide sera soumis à des forces qui provoqueront son déplacement. Pratiquement, le métal liquide circule dans un conduit aplati, généralement en acier inoxydable. Il est soumis à un champ nagnétique constant et à un courant continu de forte intensité, amenc par deux ćlectrodes en cuivre brasées de parl et d'autre du conduit (17).

Le dimensionnement optimum géométrique el électrique conduisant au rendement maximum doit tenir compte des difrérentes pertes de la machine :

- pertes électriques par effet Joule dans lo métal liquide et dans l'inducteur,

- pertes de charge hydrauliques dans le conduit.

En désignant par $l$, $e$ et $\mathrm{L}$ les trois dimensions du conduit (fig. $6 \mathrm{~b}$ ), il est facile de montrer que,

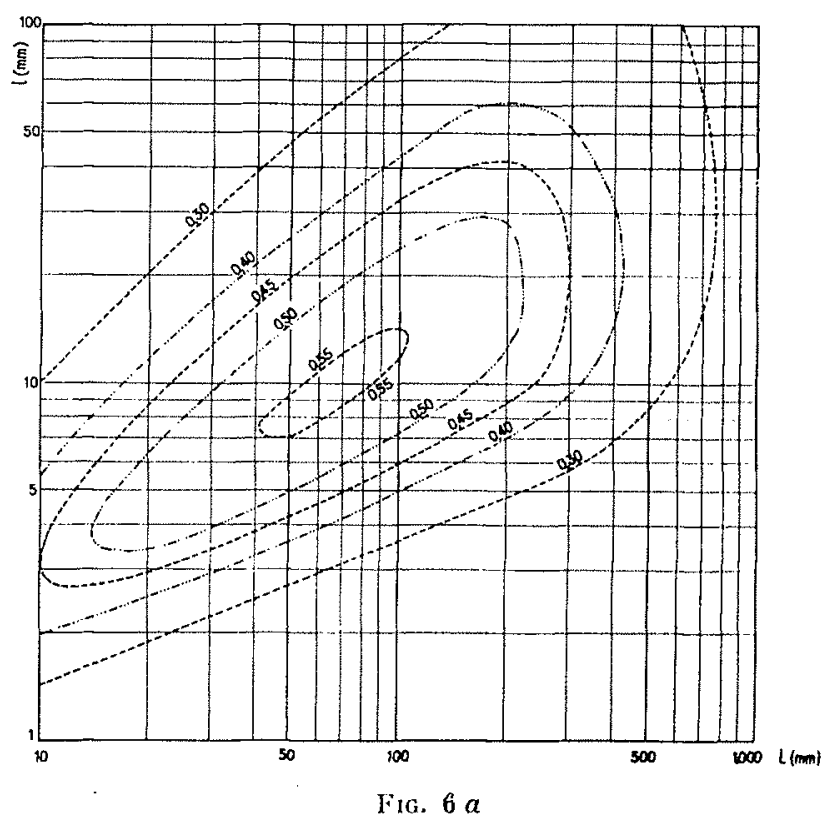

Courbes d'égal rendement théorique

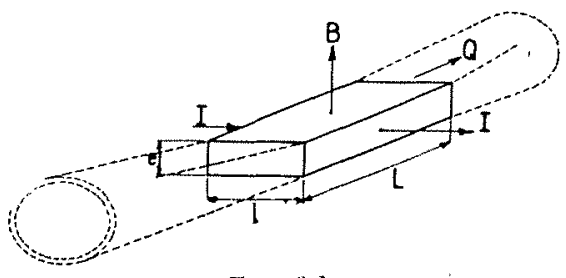

Fri. $6 b$

lompe is conduction type Faraday.

$$
\begin{aligned}
& Q=4 i) 1 / \mathrm{mll}^{\circ} \\
& p=1 \mathrm{ks} / \mathrm{cm}^{2}
\end{aligned}
$$

$e=1 \mathrm{~cm} . \quad B=4000$ gauss 
pour une différence de pression et un débit détermine, les pertes ohmiques dans le métal liquide sont proportionnelles à $l e / L$ (intérêt d'une machine longue), tandis que les pertes hydrauliques sont proportionnelles à $\left[\mathrm{L}(l+e) / l^{3} e^{3}\right]$ et sont évidemment d'autant plus faibles que la machine est plus courte.

Ces deux points de vue sont donc contradictoires et le dimensionnement optimum résultera d'un compromis, variable suivant les caractéristiques demandées à la machine. A titre d'exemple, nous donnons (fig. 6) les courbes d'égal rendement théorique d'une petite pompe de laboratoire, étudiée il $\mathrm{y}^{\prime}$ a quelques années.

Dans la réalisation de ces pompes, on doit également tenir compte de certaines pertes supplémentaires: une partie du courant d'alimentation est perdue et traverse, soit les parois conductrices de l'enveloppe, soit les parties antérieures et postérieures du métal liquide situé en dehors de la zone d'action du champ magnétique.

Une autre source de pertes supplémentaires vient de la distorsion du champ magnétique provoquée par le passage du courant de forte intensité. Cette réaction d'induit tend à augmenter le champ magnétique à l'entrée de la pompe el à le diminuer à la sortie, ce qui perturbe le fonctionnement et diminue le rendement. Pour des puissances notables, on a intérêt à compenser cette réaction d'induit, selon l'un des deux procédés suivants : une des électrodes est repliée dans l'entrefer suivant la figure, ce qui a pour inconvénient d'augmenter l'entrefer magnétique et de réduire le champ inducteur. On peut encore profiler les pôles et le conduit en augmentant l'entrefer à l'entrée de la pompe; la compensation n'est alors totale que pour un régime de fonctionnement déterminé.

Le champ magnétique inducteur est généralcment produit par un enroulement magnétisant, soit à excitation séparée (ce qui pose des problèmes d'isolement à haute température), soil à excitation série au moyen de quelques spires de cuivre non isolées.

L'intensité électrique traversant le métal liquide est de quelques milliers d'ampères pour de petites pompes dont le débit est de l'ordre de quelques $1 / \mathrm{s}$; elle est de quelques dizaines de milliers d'ampères pour des machines plus importantes; la tension d'alimentation est généraJement de l'ordre de 1 à 2 volts

Ces pompes sont donc tributaires d'une source de courant continu à forte intensité et faible tension. Cette source peut être constituée par des redresseurs, solution coûteuse, encombrante et peu efficace (rendement propre 30 à $40 \%$ ). On préfère généralement des génératrices homopolaires dont le rendement peut être élevé $(80 \%)$; la difficulté principale est la réalisation de halais collecleurs supportant des intensités de quelques dizaines de milliers d'ampères. Un joint de métal liquide (eutectique NaK par exemple) paraît donner de bons résultats. On réalise ainsi des génératrices homopolaires produisant de 20.000 à 100.000 ampères.

Le rendement global de tout le groupe de pompage varie de $15 \%$ environ pour de petites pompes, à $40 \%$ pour des installations importantes comportant une génciratrice homopolaire. Les avantages de ces pompes à courant continu sont les suivants : simplicité de construction de la pompe proprement dite, caractérisée notamment par une isolation électrique rudimentaire, possibilité d'ulilisation dans des circuits très radioactifs et à haute température $\left(800^{\circ} \mathrm{C}\right)$ avec reCroidissement de la pompe assuré seulement par rayonnement et convection naturelle. Comme inconvénient, on notera l'encombrement et le coût d'un tel groupe de pompage constitué de trois machines distinctes.

\section{C. - Pompes à conduction à courant alter- natif}

Rien ne s'oppose au fonctionnement de la pompe électromagnétique décrite ci-dessus en alimentant les ćlectrodes et l'inducteur en courant alternatif. L'effort produit est proportionnel a la valeur moyenne du produit du champ inducteur et de l'intensité électrique traversant le métal liquide; ces deux grandeurs devront être autant que possible en concordance de phase. Dans ce but, l'enroulement inducteur relié en série avec les électrodes est alimenté sous forte intensité au moyen d'un transformateur. Ce dernier peut être incorporé à la pompe elle-mème, cet ensemble étant directement alimenté par le réseau.

Par suite de courants de Foucault engendrés dans le métal liquide par le champ magnétique alternatif, ainsi que des distorsions du champ magnétique et du courant, le rendement de ces machines est généralement faible, de 10 à $20 \%$. Cependant, par suite de sa simplicité de construction, de sa facilité d'utilisation même à haute température, ce type de pompe est fréquemment utilisé dans de petites installations de laboratoire. Les caractéristiques les plus couramment réalisées correspondent à un débit de 2 à $3 \mathrm{l} / \mathrm{s}$ de sodium, dont la température peut atteindre $500{ }^{\circ} \mathrm{C}$ sous une pression de refoulement de $2 \mathrm{~kg} / \mathrm{cm}^{2}$ environ.

\section{D. - Pompes à induction}

1) GÉNÉrarités.

De même que le moleur électrique correspondant, une pompe électromagnétique à induction 
utilise les forces mises en jeu par l'action d'un champ magnétique alternatif nobile, sur un conducteur. On sait, en effet, que le déplacement d'un champ magnétique engendre par induction des courants électriques dans le conducteur; la réaction de ces courants et du champ donnent lieu à des forces électromagnétiques tendant à entrainer le conducteur dans le sens du champ (18).

Une pompe électromagnćtique asynchrone peut ainsi se schématiser par la ligure 7 . Cette figure n'est autre que le développement sur un plan d'un moteur asynchrone dont l'induit, ana-

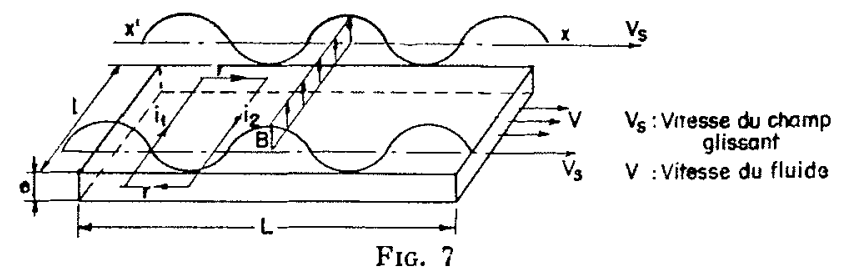

principe de fonetionnement des pompes électromagnétiques à induction.

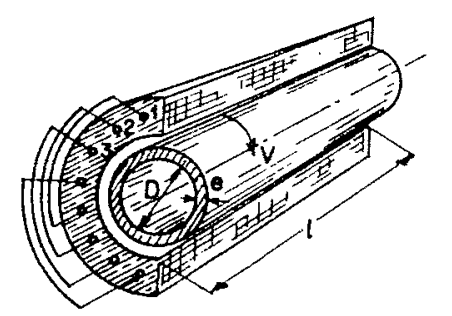

Fig. $y$

Moteur asynchone triphasé it rotor massif'.

losue à une cage d'écureuil, serait, en fait, constilué d'un lube d'épaisseur $e$, de longhueur $l$ et de longueur circonférenticlle $L=\pi$ I) (fig. 8). L'inducteur du moteur asynchrone développé cngendre, au lieu du champ toumant habituel, un champ sinusoïdal $B$ se déplaçant à la vitesse $V_{s}$ le lons de l'axe $x^{\prime} x$. L'induit du moteur est remplacé par le liquide conducteur contenu dans l'entrefer d'épaisseur $e$, de largeur $l$, de longueur L. Dans ce liquide prendront naissance des courants $i_{1}, i_{2}$ analogues à ceux qui traversent les barres d'un rotor en cage d'écureuil, el par réaction électromagnétique, le conducteur liquide sera soumis à un effort qui tendra ì le déplacer à une vitesse $V$ inférieure à la vitesse $V_{s}$ du champ glissant. Un parametre essentied de fonctionnement est lo glissement $g$ egal à la différence relative $\left(\mathrm{V}_{s}-\mathrm{V}\right) / \mathrm{V}_{s}$.

a) Une pompe à induction est couramment réalisée sous une forme correspondant directement au schéma de principe; il s'agit de la pompe plate représenléc sur la figure 9. Un conduit métallique aplati est alors placé entre deux armatures magnétisées par un enroulement électrique logé dans des encoches et alimenté cu

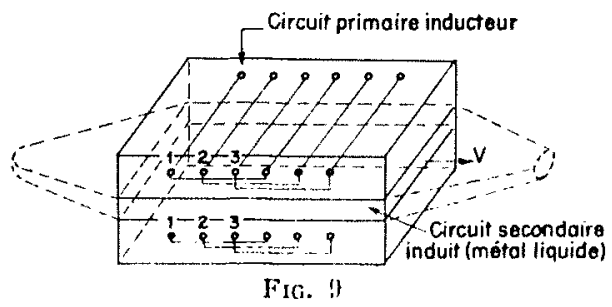

Pompe electromagnélique "plate ».

courant triphase. Deux barres d'un métal bon conducteur sont brasées de part et d'autre du conduit pour réduire la résistance opposće à la fermeture des couranls induits (19).

Ce type de pompe (fig. 10 et 11) est assez lar-

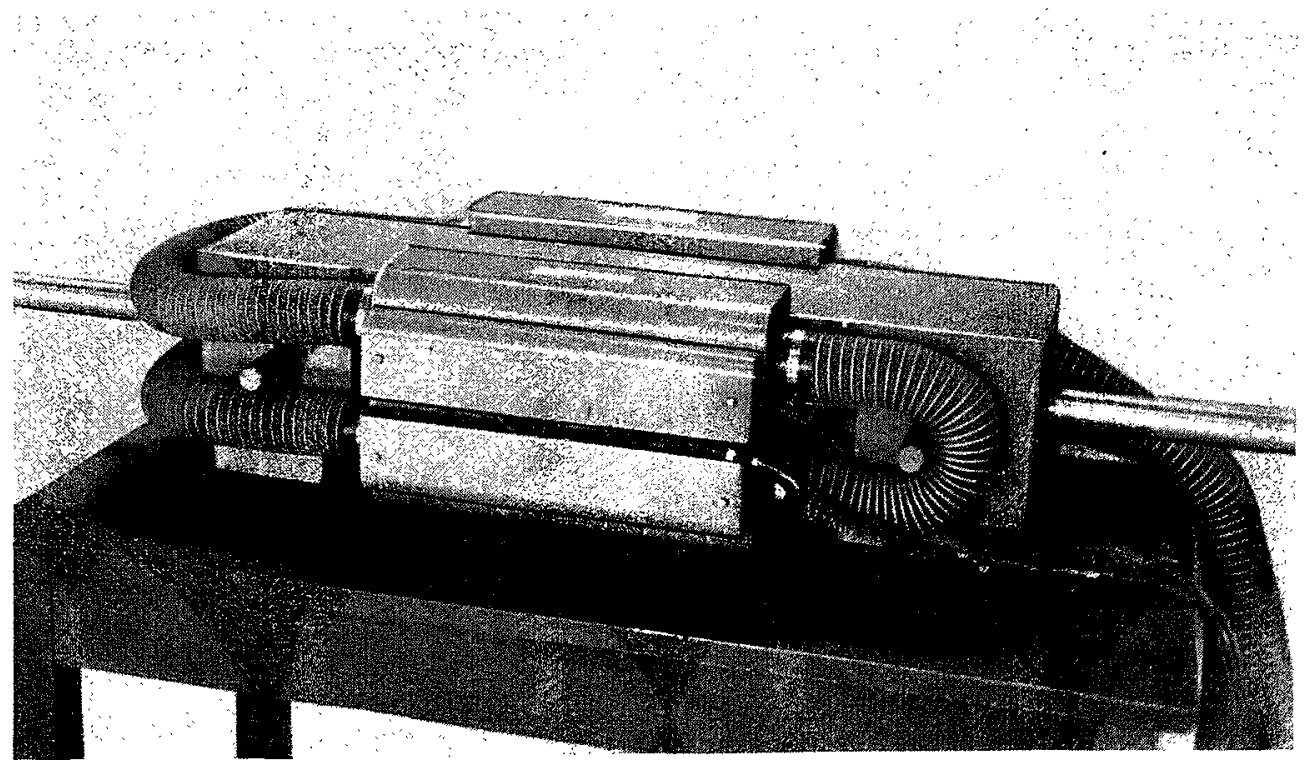

I.xis. 10

Pompe électromagnétique plate E.D.F. 
gement lépandu et convient en particulier si le rapport Débit/Pression doit être relalivement élevé. Une pompe de ce type, ainsi que du type suivant, a été étudiée sous la conduite de M. Remenierts, Chef du Service des Eudes Hy-

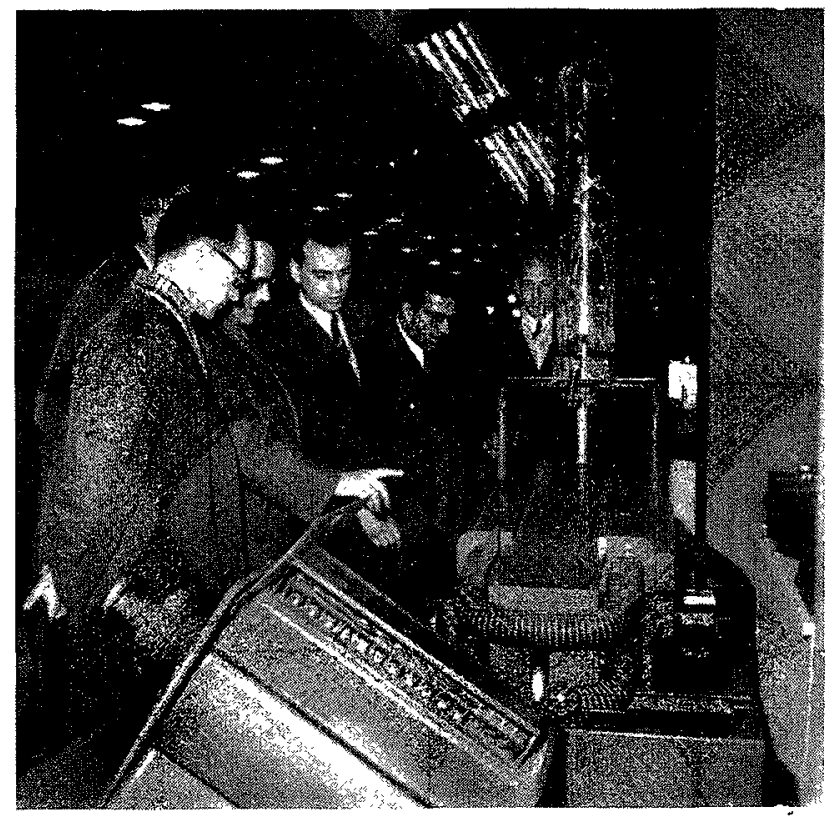

FIG. 11

Pompe électromagnétique plate E.D.F. fonctionnant avec du mercure (Exposition anmuelle de la Société Française de Physique, avril 1956).

drauliques par le Département Essais Extérieurs du Service des Essais et Laboratoires Hydrauliques de l'Electricité de France. Cette pompe est actuellement en cours d'essai de fonctionnement avec alliage sodium-potassium, au Commissariat à l'Energie Atomique.

b) Une des transformations que l'on peut faire subir au schéma de principe 7 consiste à courber la largeur $l$ du conduit suivant une circonférence, refermée sur elle-même. On obtient ainsi la pompe annulaire, connue sous le nom de pompe Einstein-Szilard (20) (fig. 12 et 13). Les dimensions caractéristiques de la veine liquide

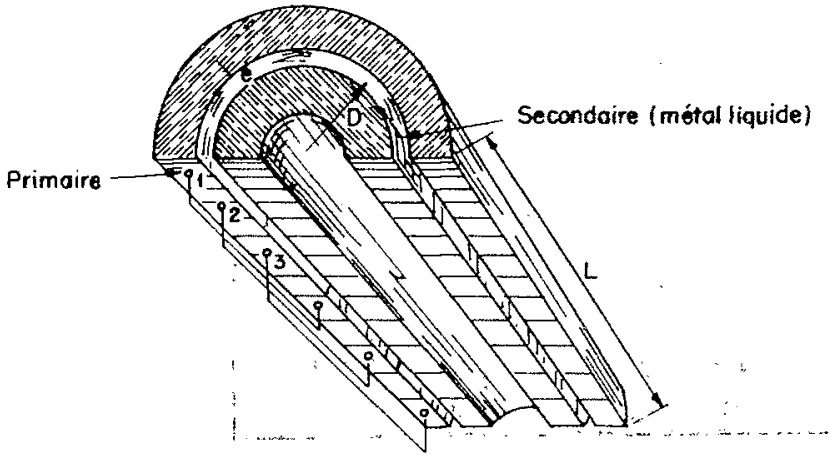

Fig, 12

Pompe électromagnétique " annulaire ». sont: longueur $\mathrm{L}$, largeur $l=\pi \mathrm{D}$, épaisseur $e$; l'inducteur est alor's constituć d'une série de galettes circulaires alimentées en courant triphasé; les courants induits décrivent également des spi-

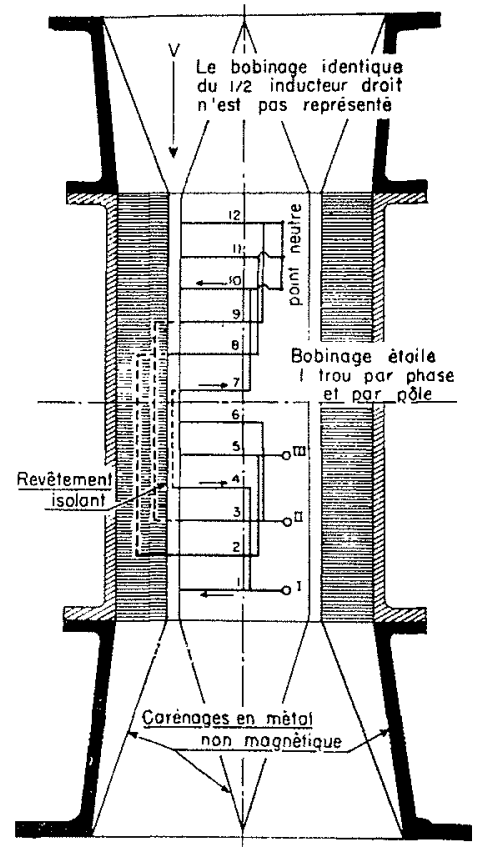

Frg. 13

Pompe électromagnétique annulaire (figure extraite d'une communication de M. G. Remenieras publiee en 1948).

res circulaires centrées sur l'axe el se referment donc directement sur eux-mêmes, sans résistances de contact supplémentaires. Une pompe de ce type a également été éludiée (fig. 14); des difficultés de réalisation ont retardé son développement et on a encore relativement peu d'expérience concernant son fonclionnement.

c) Une autre transformation consiste à courber la longueur $\mathrm{L}$ du schéma de principe 7 de manière à l'enrouler suivant une hćlice de pas égal à la largeur du conduit $l$. Dans cette pompe

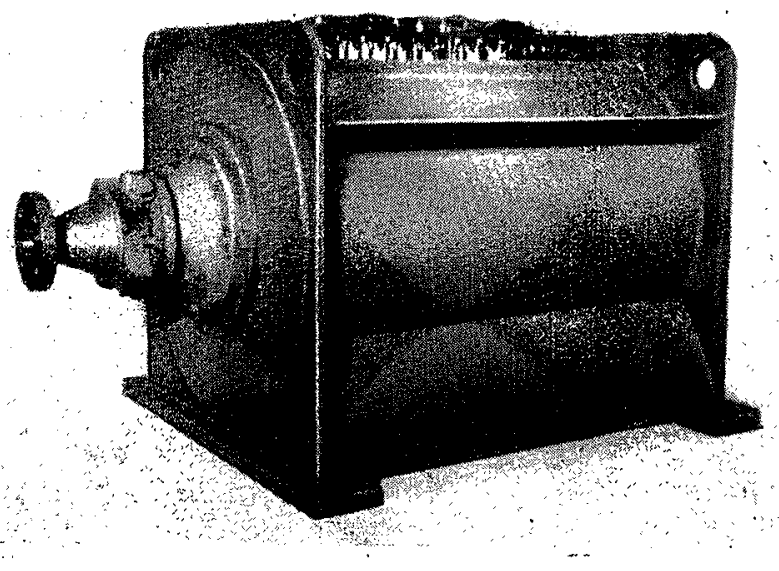

Fig. 14

Pompe électromagnétique annuaire E.D.F. 
hélicoïdale, on retrouve alors le stator d'un noteur asynchrone figuré par les conducteurs 1 , 2 , 3, etc. traversés par des courants polyphasés (fig. 15). Par l'effet du champ tournant, le li-
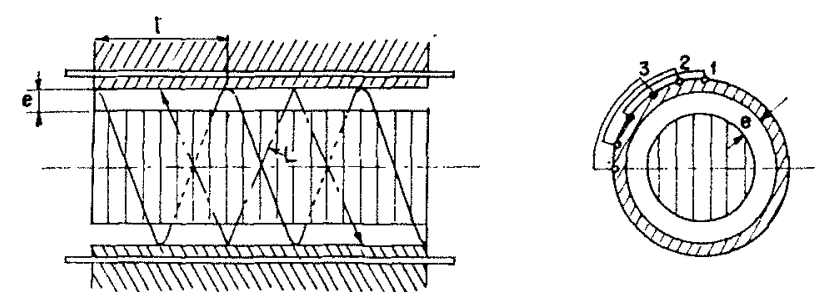

F1G. 15

Pompe électromagnètique « hélicö̈dale ».

quide conducteur décrit une hélice de longueur totale $\mathrm{I}$, en traversant une conduite de section droite rectangulaire de largeur $l$ et d'épaisseur $e$; une telle disposition est utilisée pour accroître le rapport $\mathrm{L} / l$ sans trop allonger le circuit inducteur; elle permet ainsi d'obtenir un rapport Pression/Débit important. Les pertes dues à la composante axiale $d u$ déplacement du métal liquide ne paraissent pas négligeables; de plus, la réalisation du conduit de cette pompe présente certaines difficultés.

A l'actif de ces différents types de pompes à induction, on relève l'alimentation directe à partir des sources conventionnelles d'énergie, les problèmes caractéristiques de ce type de machine. Au-delà de $400^{\circ}$, le dimensionnement de la machine doit en tenir compte, et s'ecarter ainsi de l'optimum.

\section{2) Consmérations thiÉorioues.}

Le fonctionnement électrique de la pompe à induction peut être analysé de la même manière que celui d'un moteur asynchrone; il est également commode de représenter électriquement la machine par son schéma équivalent (fig. 16), qui groupe des impédances calculables en fonction du dimensionnement géométrique et électrique de la pompe.(21).

Pour un type de fabrication déterminé, le dimensionnement géométrique de la pompe à induction est en fait essentiellement défini par la longueur du double pas polaire $\mathrm{L}_{\mathrm{r}}$ (Iongueur d'onde), et l'épaisseur de la veine liquide $e$; quant au dimensionnement électrique, deux paramètres classiques suffisent dans une certaine mesure : charge linéique $(A)$ et densité de courant $(\delta)$ dans l'inducteur.

La pompe à induction présente néanmoins par rapport au moteur asynchrone quelques particularités qui rendent son analyse beaucoup plus complexe.

Tout d'abord, la technique usuelle des courants filiformes ne peut être appliquée au cir-

Quadripole equivalent à un moteur osynchrone

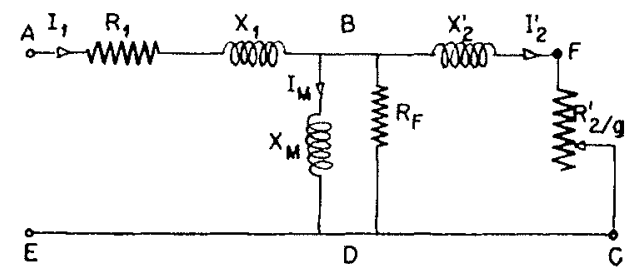

Rt : Résistance du primaire,

$\mathrm{X}_{1}$ : Réactance de dispersion du primaire,

$\mathrm{X}_{\mathrm{y}}$ : Réactance de magnétisation,

$R_{\mathrm{F}}$ : Résistance de peries dans le fer,

Quodripole équivalent ò une pompe asynchrone

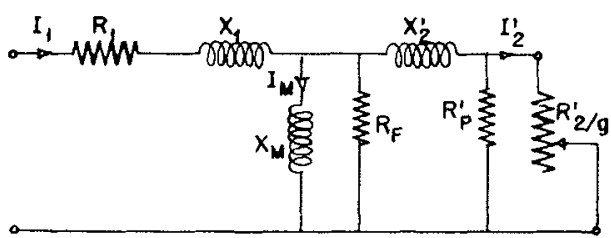

$X_{\prime 2}^{\prime}$ : Réactance de dispersion da secondaire

$R_{2}^{\prime}$ : Résistance du secondaire

$\mathrm{R}^{\prime}{ }^{\prime}$ : Résistance des parois

j ramenes

au

$I_{1}$ : Intensité primaire,

$I_{M}$ : Intensité du courant magnélisant,

$I_{2}:$ Intensité dans le circuit secondaire lamenée at primaire.

FIG. 16

Quadripoles équivalents à un moleur et à une pompe asynchrone.

courants de forte intensité nécessaires étant alors produits directement par induction dans le liquide. Les caractéristiques des divers modèles réalisés s’échelonnent de quelques. $1 / \mathrm{s}$ à $200 \mathrm{l} / \mathrm{s}$ sous des pressions de refoulement atteignant $7 \mathrm{~kg} / \mathrm{cm}^{2}$; le rendement atteint $40 \%$ environ pour les machines les plus importantes. L'échauffement des enroulements inducteurs, nécessairement placés au voisinage immédiat du métal liquide porté à haute température, est un des cuit secondaire massif (métal liquide), où la direction des courants électriques n'est pas définie, a priori. De plus, le champ magnétique présenle, en général, des discontinuités à l'entréc et à la sortie de la pompe, qui entrainent l'apparition d'harmoniques dans l'équation du flux magnétique; leur limitation est essentielle au bon fonctionnement de la machine.

Comple tenu de ces facteurs, l'étude du schéma équivalent permet de calculer, en fonc- 
tion du dimensionnement, les caractéristiques électriques de fonctionnement (pression théorique, débit, rendement électrique); il s'agit ensuite d'introduire les pertes de charge hydraulique de la pompe, dont l'importance est notable bal, puissance spécifique) en fonction du glissement (paramètre de fonctionnement) et pour un certain dimensionnement électrique $(A, \delta)$ et micanique $\left(\mathrm{L}_{\mathrm{P}}, e\right)$. Les formules obtenues, commodes d'emploi pour un avant-projet de pompe,

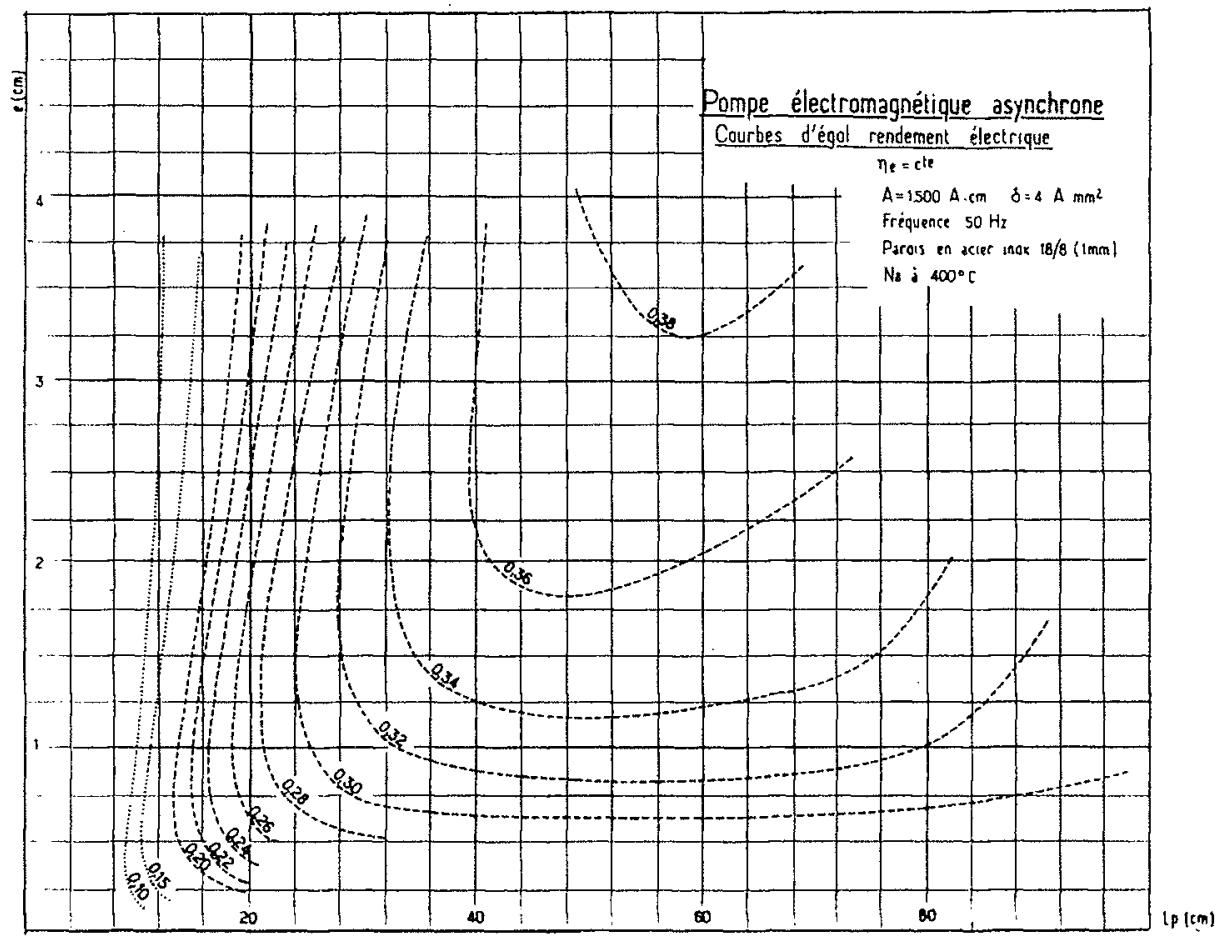

Fig. 17 Courbes d'égal rendement électrique.

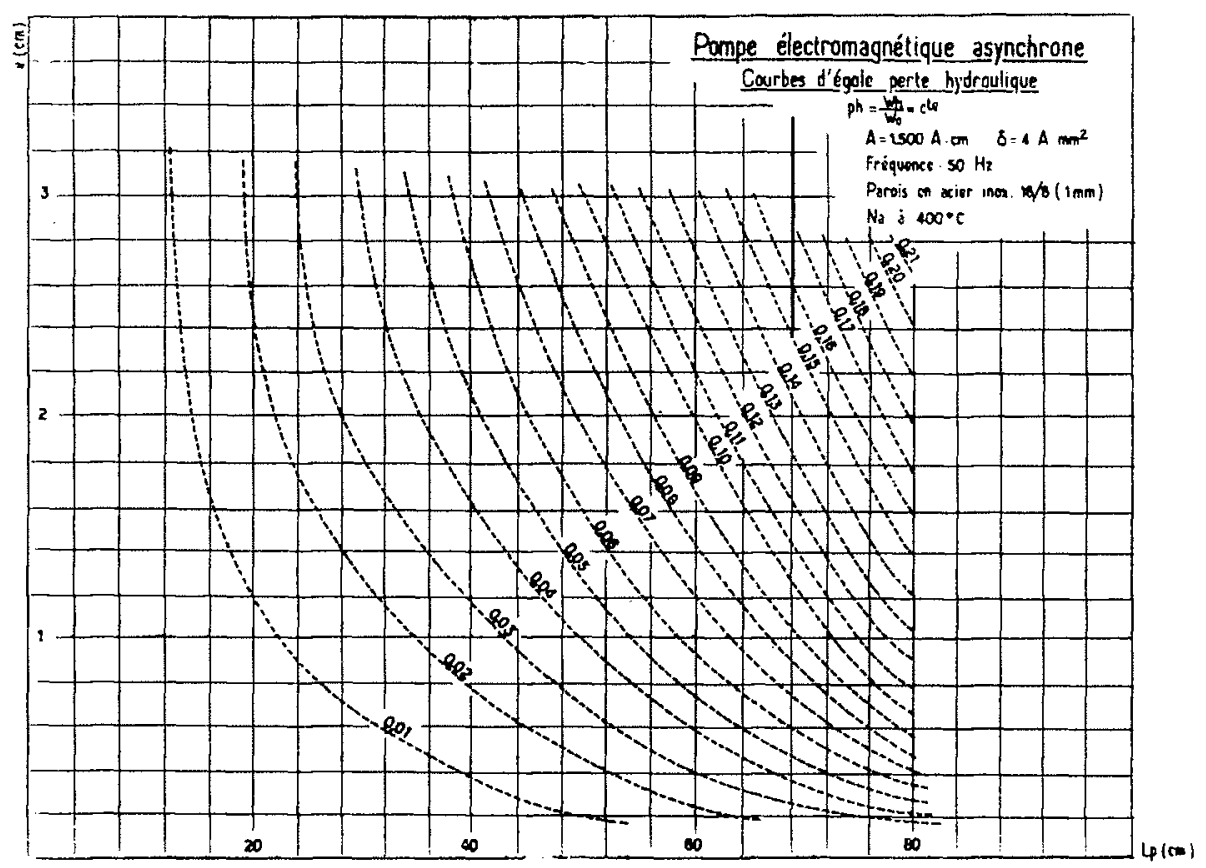

Fig. 18 Courbes d'égale perte hydraulique.

aux vitesses élevées - quelques $\mathrm{m} / \mathrm{s}$-- que doit atteindre le métal iiquide.

On obtient ainsi les caractéristiques finales de la machine (pression netle, débit, sendement glo- sont plus générales que celles utilisées habituellement pour le calcul des moleurs électriques. Dans ce dernier cas, en efiet, divers élénents sont connus initialement, d'après l'expérience 
que l'on a de ces machines, le projet d'un nouveau moteur consistant le plus souvent à.interpoler entre les réalisations précédentes.

D'après l'application numérique de ces formules à un cas particulier, une pompe électro- sous forme de pertes par courants de Foucault dans le métal liquide et dans les parois métalliques du conduit; les pertes de charge hydrauliques représentent également une part notable de l'énergie perdue.

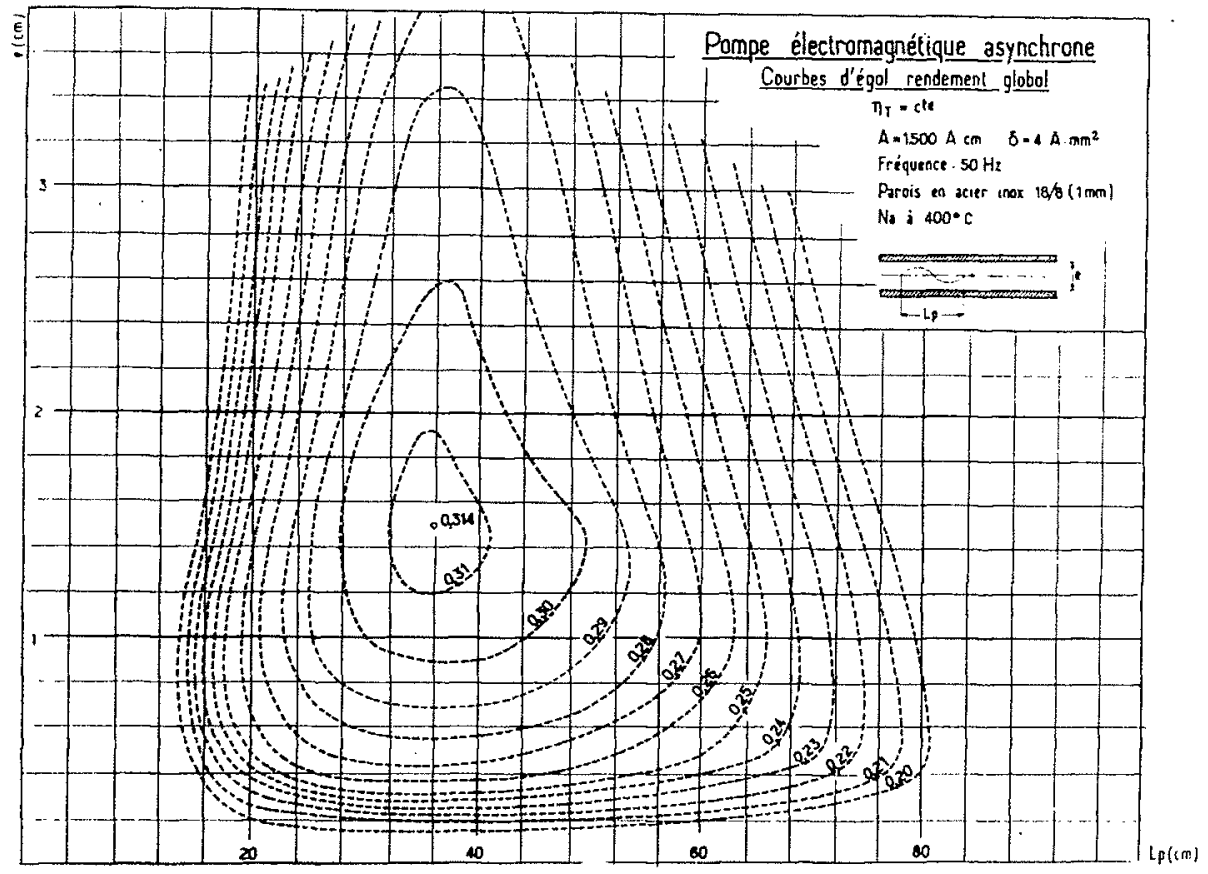

Iivi. 19

Courbes d'egat rendement global.

FIG. 20

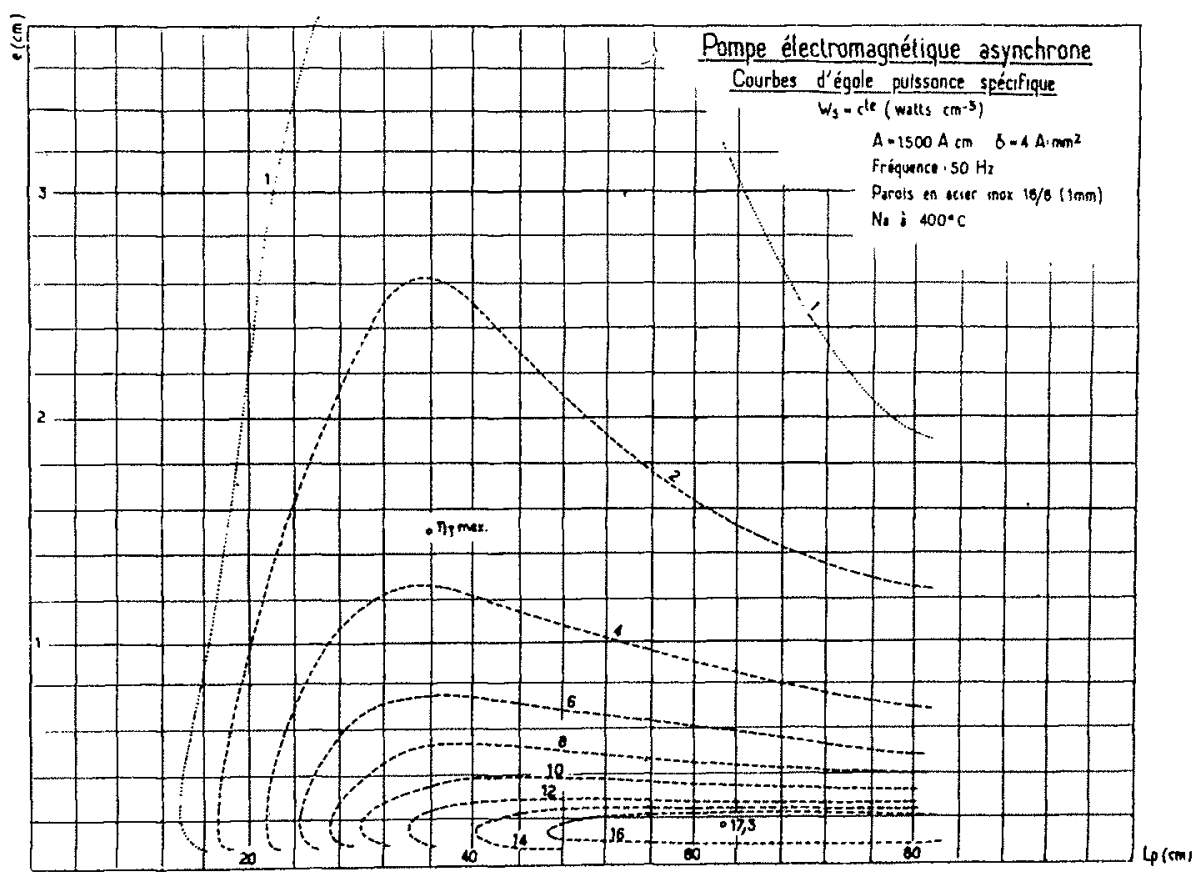

magnétique présenterait, à dimensionnement équivalent, des caractéristiques très médiocres par rapport à celles d'un moteur asynchrone de type courant : rendement réduit au cinquieme, puissance massique treize fois plus faible. L'énergie perdue est principalement dissipcée
Ce calcul, effectué dans le cas particulier du dimensionnement d'un moteur asynchrone classique, est ensuite généralisé, de manière à fixcr le dimensionnement optimum de ces pompes, qui se présente en fait comme un compromis entre exigences contradictoires d'ordre électrique 
et hydraulique. Pour chaque dimensionnement on ne retient que les caractéristiques correspondant au rendement maximum, et on en trace les courbes d'égale valeur en fonction de deux des paramètres cités $\left(\mathrm{L}_{1}, e, \mathrm{~A}, \delta\right)$. maximum serait ainsi de $32 \%$ environ et la puissance spécifique maximum de $15 \mathrm{~W}: \mathrm{cm}^{3}$ (approximativement moilié de celle d'un moteur asynchrone) $\left(^{\star}\right)$. Il importe de noter que certaines hypothèses de calcul sont, dans une cer-

Fig. 21

Courbes d'égal rendement global
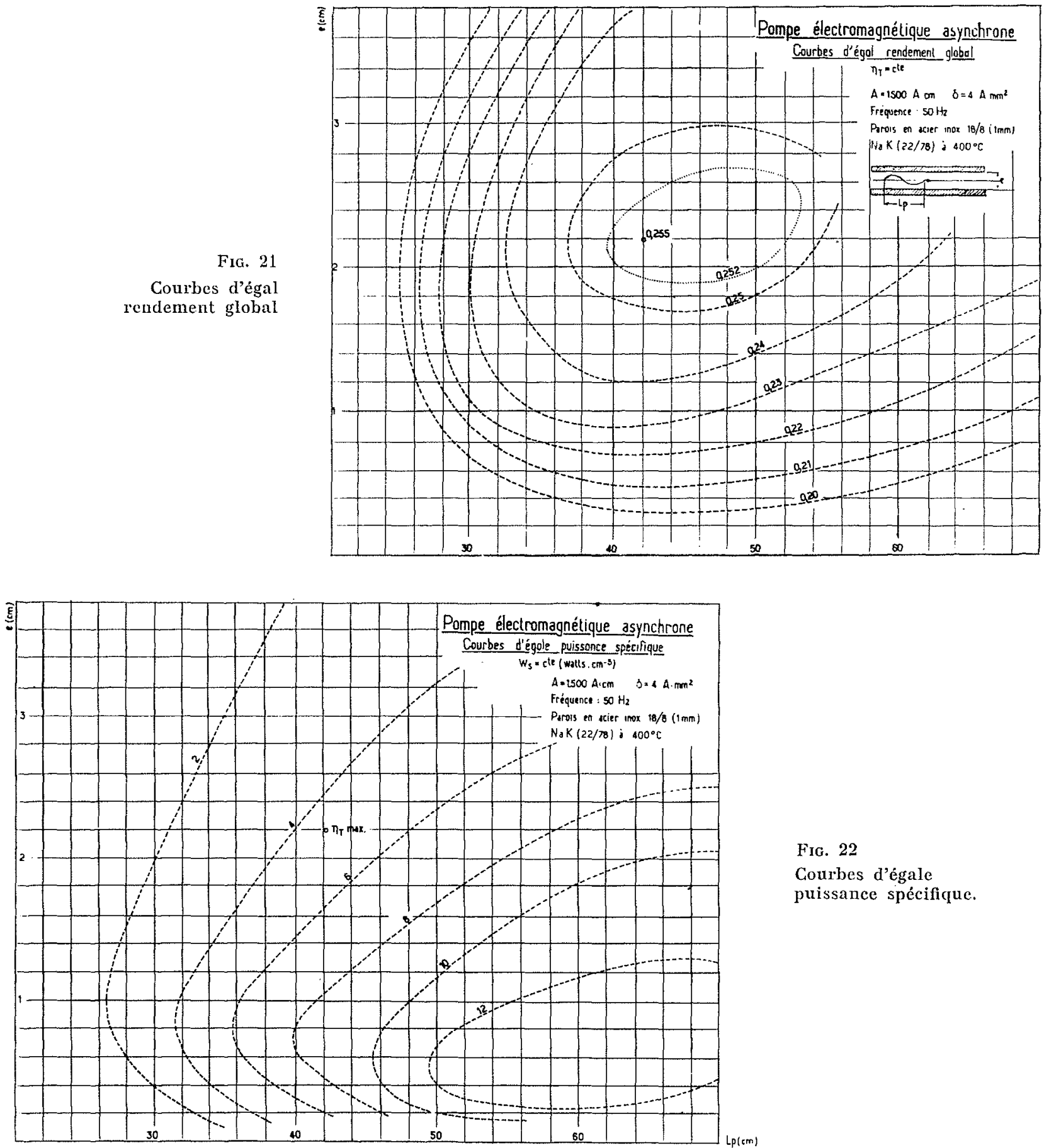

FIG. 22

Courbes d'égale puissance spécifique.
Nous donnons en particulier, figures 17 à 20 , les caractéristiques calculées dans le cas d'utilisation du sodium (pompe alimentée en $50 \mathrm{~Hz}$, parois en acier inoxydable $18-8, \mathrm{~A}=1.500 \mathrm{~A}$ : $\left.\mathrm{cm}, \delta=4 \mathrm{~A}: \mathrm{mm}^{2}\right)$. On voit que le rendement taine mesure, fonction de la puissance de la machine que l'on a supposée être relativement

(*) On désigne ici par puissance spécifique la puissance utile ramenée à l'unité de volume du circuit inducteur. 
faible (quelques $\mathrm{kW}$ ); le rendement augmenterait en fait légèrement avec les dimensions de la machine. En outre, ce calcul effectué en accord avec nos premiers essais, ne tient pas très marquée sur les caractéristiques optima possibles. Les figures 21 à 24 correspondent au cas d'un alliage $\mathrm{NaK}$ et de l'eutectique $\mathrm{Pb}-\mathrm{Bi}$, métaux liquides qui, avec le sodium, sont parmi

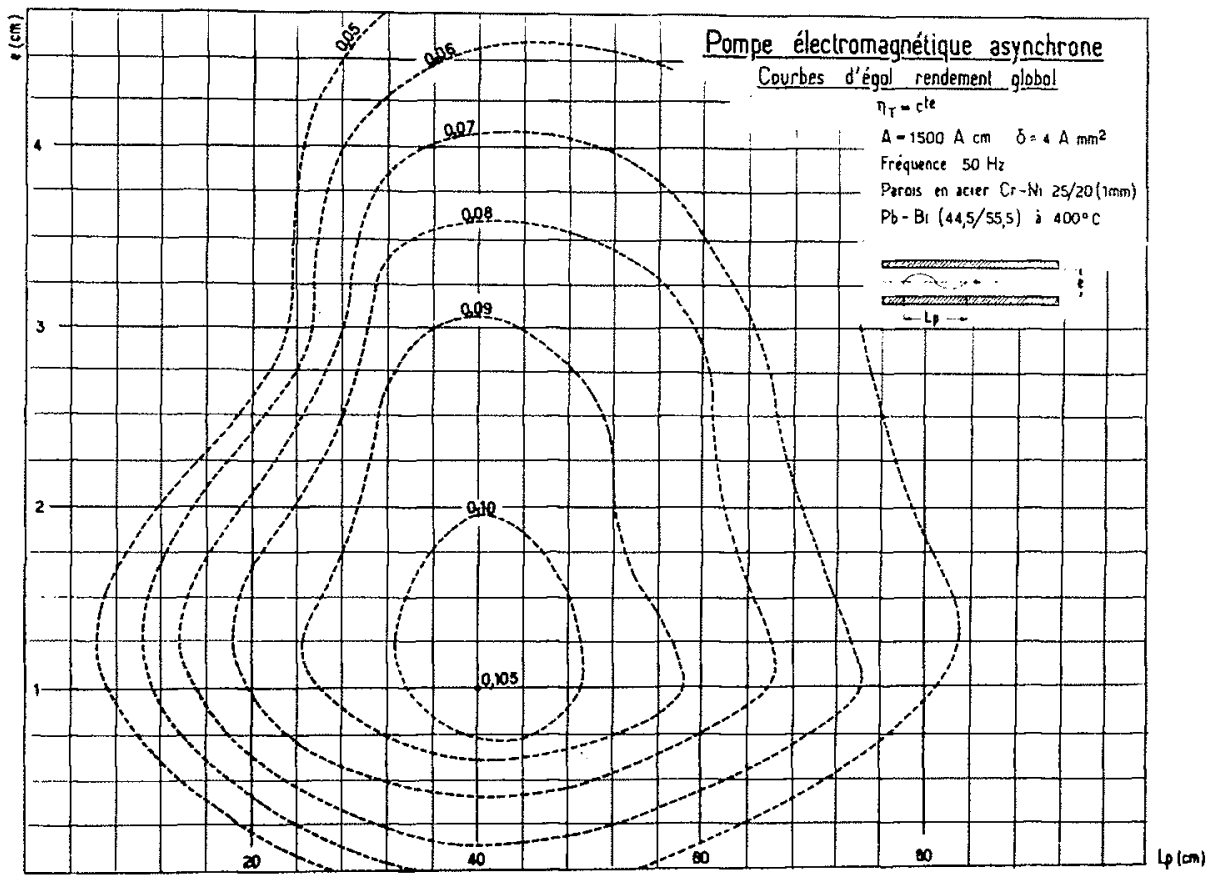

FII., 23

Courbes d'égal rendement global.

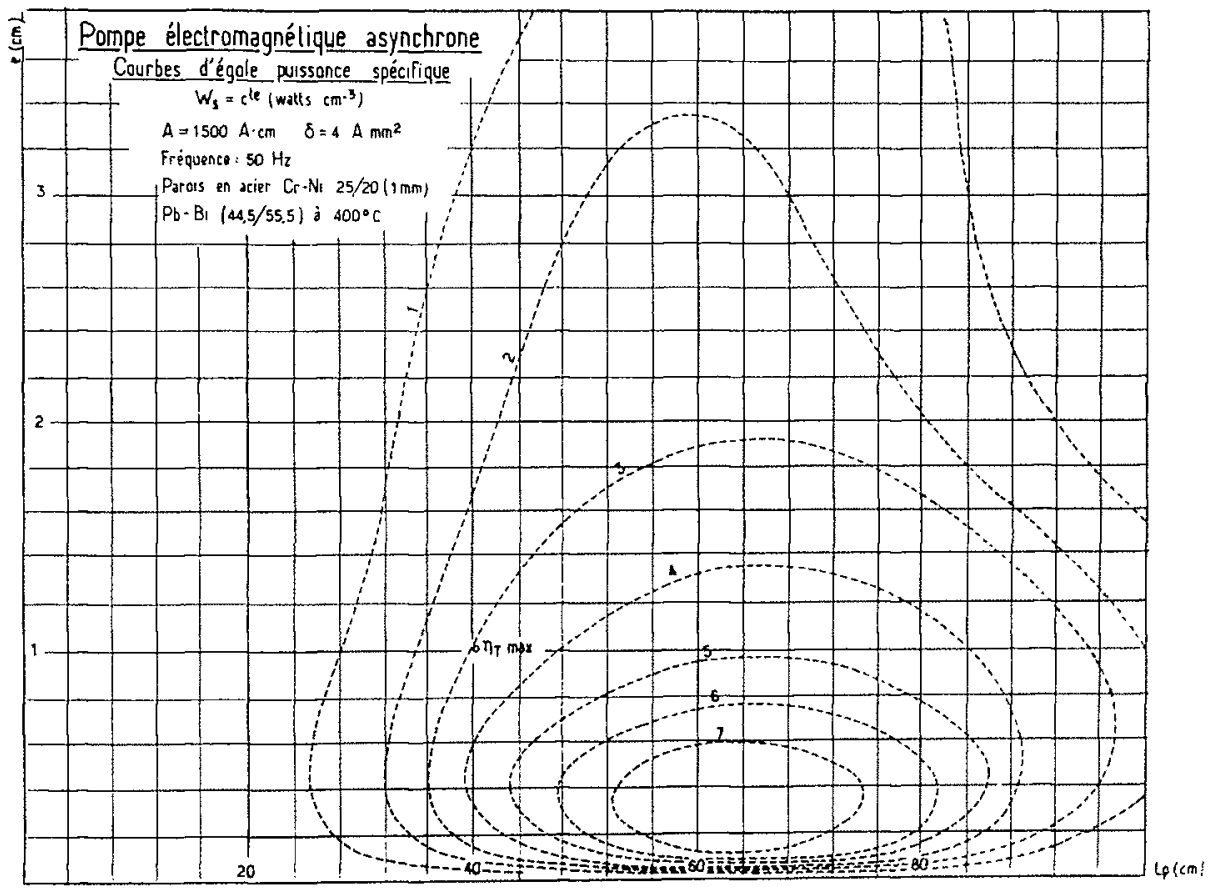

Frg. 24

Courbe d'égale puissance spécifique.

compte de la compensation des pertes importantes dues aux effets d'extrémité.

La résistivité électrique et le poids spécifique du métal liquide utilisé ont une influence les plus intéressants au point de vue transfert thermique.

Même avec le sodium qui, parmi les mitaux liquides à basse température, a la résistivité la 
plus faible, les performances ne deviennent comparables à celles des moteurs électriques classiques que si la pompe est alimentée sous fré- quence réduite, et si le métal liquide circule dans un conduit isolant, solutions par ailleurs très discutables.

\section{IV. - COMPARAISONS ENTRE L.ES DIVERS TYPES DE POMPES A METTAUX LIQUIDES}

On peut compléter cel exposé par une comparaison indiquant dans quelle mesure les qualités exigées sont remplies par chaque type de pompe. Cette comparaison est schématisée dans le tableau ci-contre (fig. 25), sous forme d'une «feuille de notation » présentant nécessairement un certain arbitraire. Ce tableau appelle les remarques suivantes: quide; certaines de ces pompes sont, à titre de sécurité, entièrement enfermées dans un épais carter plus résistant.

Des réserves sont à faire, au sujet de la résistance à la corrosion de la totalité des matériaux constituant les pompes mécaniques. L’organe susceptible d'être attaqué est, suivant le cas : le manchon refroidi, les paliers ou le joint soumis

Fig. 25. - Comparaison ENTRe les types principaux de pompes a métaux liduides

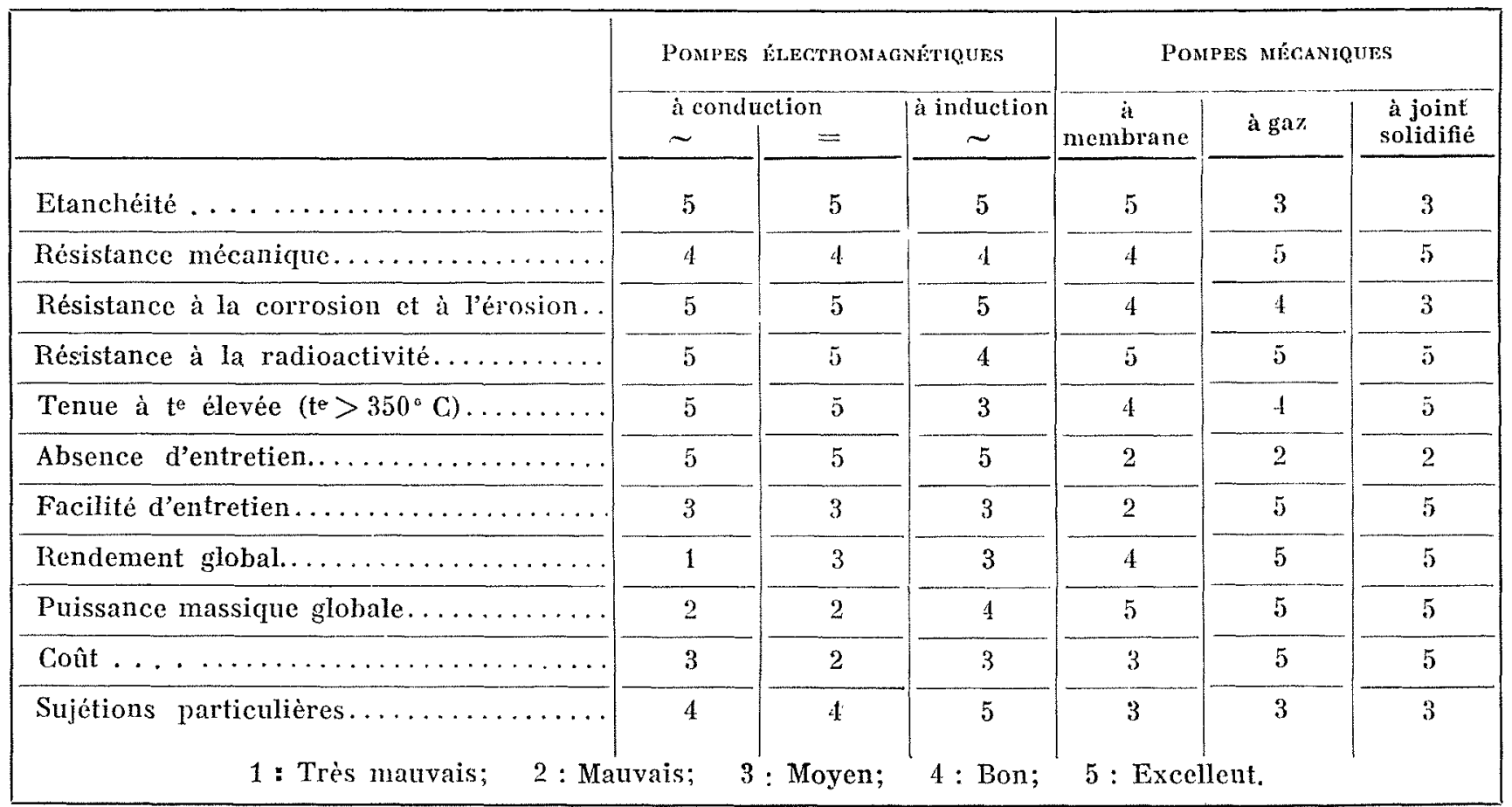

L'étanchéité n'est véritablement absolue que dans les pompes entièrement fermées, sans aucun joint, et à couple transmis électromagnétiquement à travers une membrane mince. Si l'étanchéité des pompes mécaniques à couverture gazeuse et à joint de sodium solidifié paraît correcte, elle est néanmoins à la merci d'une avarie du dispositif assurant cette étanchéité.

On peut de même associer les pompes électromagnétiques et la pompe à rotor immergé en ce qui concerne leur résistance mécanique; la présence d'une membrane métallique mince soumise à la pression est un point de moindre résistance dans l'ensemble du circuit de métal li- aux vapeurs métalliques. Dans les deux derniers cas, cette attaque s'aggrave à température élevée.

L'utilisation des métaux liquides radioactifs à température supérieure à $350{ }^{\circ} \mathrm{C}$ est, par ailleurs, une des principales difficultés de réalisation des pompes à induction, coont l'inducteur est difficilement protégé de ces flux de radiations.

Le problème de l'entretien ne se pose que pour les pompes mécaniques; il est aisé à lésoudre, dans le cas des pompes à couverture gazeuse et à joints solidifiés; la présence de soudure 
d'étanchéité le rend moins facile pour une pompe à rotor immergé.

Les rendements les plus élevés sont ceux des pompes mécaniques à couverture gazeuse et à joints solidifiés (75\%), à rotor immergé $(60 \%)$. Viennent ensuite les pompes électromagnétiques dont les rendements atteindraient les ordres de grandeur suivants en cas d'utilisation du sodium : pompe à courant continu : $50 \%$; pompe à induction : $40 \%$; pompe à courant alternatif : $15 \%$.

Le faible rendement de ce dernier type, excellent à d'autres points de vue, parait devoir limiter son utilisation au domaine du laboratoire. Pour les autres types de pompe, et malgré la variation notable que.l'on constate sur les rendements, on ne doit pas perdre de vue le peu d'influence qu'elle présente sur le bilan général d'un équipement de production d'énergie d'origine nucléaire.

Les pompes électromagnétiques sont moins bien adaptées que les pompes mécaniques en ce qui concerne le pompage des métaux liquides lourds et de forte resistivité (bismuth, plomb). En effet, une pompe électromagnétique n'engendre pas «une hauteur manométrique», mais un effort fonction inverse de la résistivité du métal liquide; de plus, à la différence de pression correspondante doit être retranchée une perte de charge proportionnelle au poids spécifique.

La comparaison des coûts relatifs par unité de puissance liée, dans une certaine mesure, à la puissance massique, est assez approximative. Les pompes mécaniques, à couverture gazeuse et à joints solidifiés, viennent actuellement en tête, ce qui, dans plusieurs cas, les a fait préférer.

Enfin, des sujétions particulières à un type de pompe présentent, parfois, une importance primordiale. C'est ainsi que la pompe à couverture gazeuse doit comprendre un système de contrôle du niveau supérieur du métal liquide, contrôle délicat, surtout si le régime de fonctionnement est très variable. La pompe à joints solidifiés ne convient que si le liquide esi parfaitement pur et ne contient aucune suspension solide.

\section{V. - CONCLUSION}

La technique des métaux liquides est encore beaucoup trop récente pour qu'il soit possible de terminer cet exposé par une conclusion favorable à tel ou tel type de pompe. On a seulement voulu montrer le caractère très relatif du choix d'une pompe à métaux liquides, choix essentiel- lement fonction du problème particulier à résoudre, dans un état donné de la technique.

En terminant, nous tenons à remercier tous ceux qui nous ont aidés dans ces travaux, et particulièrement le Groupe d'Etude des métaux liquides du Commissariat à l'Energie Atomique.

\section{BIBLIOGRAPHIE}

(1) Vautney (L.). - Utilisation des métaux liquides dans les réacteurs nucléaires. Cours de génie alomique, Centre d'études nucléaires de Saclay.

2) Lyon, R. N. (Ed.). - Liquid Metals Handbook, Navexos P 733, June 1952. A tomic Energy Commission. Department of the Navy, Washington D.C.

(3) Carey, B. Jackson (Ed.). - Liquid Metals Handbook. TIB 5277, July 1955. Atomic Energy Commission, Department of the Navy, Washington D.C.

(4) Banister (C.) et al. - A report on the proceedings of the liquid metal utilisation conference, May 1953, AERE X/R 1381.

(5) Trocki (T.) et al. - Sodium and sodium-potassium alloy for reactor cooling and steam generation. Rapport présenté da la Conférence Internationale sur l'utilisation de l'énergie atomique à des fins pacifiques, Genève 1955. P. 123, U.S.A.

(6) Barnes (A.H.). - Pumping of liquid metals. Rapport présenté da la Conférence Internationale sur l'utilisation de l'énergie atomique à des fins pacifiques, Genève 1955. A/Conf. $8 / \mathrm{P} / 121$, U.S.A., 30 June 1955.

(7) Northrup (E. F.). - Trans. Amer. Electro Chem. Soc, vol. 15, 1909.
(8) Chums (L. W.). - Vacum pump. U.S. Patent spec. 1,298,664, April 1919 .

(9) Banbridge (K. T.). - Liquid conductor pump. U.S. Patent spec. 1,660,407, February 1928 .

(10) Remeneras (G.). - Sur la possibilité de transformer directement en énergie électrique une partie de l'énergie d'une veine fluide. La Houtle Blanche,

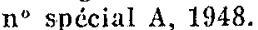

(11) CaGE (J.F.). - Electromagnetic pumps for high temperature liquid metals. Mechanical Engineering, June 1953 , p. 467.

(12) BnuL (E. F.). - Development of special pumps for liquid metals. Mechanical Engineering, mai 1953, p. 369 .

(13) Watr (D. A.). - Electromagnetic pump for liquid metals. Engineering, April 27, 1956.

(14) Blake (L. R.). - Conduction and induction pumps for liquid metals. Proceedings of the institution of electrical engineers, 1956, vol. 104, Part A.

(15) Hartmann (J.), Lazants (F.). - Experimental investigations of the flow of mercury in a homogeneous magnetic field. Det $\mathrm{Kgl}$. Danske Videnskabernes Selskib 1937,15, p. 7 . 
(16) Murgatroyd (W.). - Experiments on magnetohydro-dynamical channel flow. Philosophical Magazine, 1953,44, p. 1248 .

(17) Barnes (A.H.). - Direct current electromagnetic pumps. Nucleonics, June 1953, p. 16.

(18) WatT (D. A.). - A study in design of travellingfield electromagnetic pumps for liquids metals. AERE ED/D 1696.
(19) English Electric Company, Ltd. - Improvements relating to Electro-hydraulic Apparatus. U.K. Patent Specif. 645,881, April 22, 1948.

(20) Ernstrin (A.), Szilaind (L.). - Pump especially for refrigerating machines. U.K. Patent Specif. 34A,881, Dec. 3, 1929 .

(21) Colurvs (G.D.). - Operation and analysis of a 100 P.S.I. Electromagnetic pump. KAPL 668, January $19,1952$.

DISCUSSION

Président: M. Remenienas

M. Le Président remercie M. Hermant de son expose particulièrement dair qui témoigne de sa virtuosité dans un domaine nouvean; son travail évitera de recherchel dans de nombreuses revues, surtout étrangères, une documentation qu'il est toujours pénible, non seulement de trouver, mais aussi d'assimiler.

M. Darrieus demande si, dans les pompes électromagnétiques à eonduction utilisant du mercure, des difficultés n'ont pas élé constatées du fait de la résistance de passage du courant entre le fer et le mercure, comme cela a été observé il $y$ a quelques années dans des machines unipolaires, pour lesquelles, il est vrai, cetle résistance de passage disparaissait brusquement au-dessus de $200^{\circ}$, probablement paree que le mercure mouille le fer au-delà de cette température; ce qui peut d'ailleurs faire craindre une corrosion comme avec les métaux qui, mouilles par le mercure, s'amalgament spontanément à froid.

M. Heruant répond qu'il y a des pertes électriques au contact entre liquide et paroi, principalement dans les pompes à conduction qui sont traversées par des courants électriques intenses. Dans le cas du sodium, Ia résistance de contact à la paroi est faible, du moins a ux températures élevées où il est normalement ulilisé.

M. Hupner ayant demandé si la pression a une intluence pratiquement négligeable sur le point de fusion du sodium, M. Henmant répond affirmativement.

M. Schlag demande si, pour les pompes mécaniques, il faut, dans l'établissement des courbes caraetéristiques, tenir compte de l'influence du nombre de Reynolds, ou si cette influence est négligeable.

M. Hermant répond que dans le cas du sodium, le poids spécifique est proche de celui de l'eau et la viscosité absolue est de l'ordre du tiers, ver's $300^{\circ} \mathrm{C}$. De co fait, le nombre de Reymolds est un peu différent.

A propos de l'intervention de M. Hupner, M. le Président remarque que si les pompes à métaux liquides intéressent aujourd'hui presque uniquement les réacteurs nucléaires, elles peuvent trouver, dans l'avenir, d'autres applications utilisant les propriétés exceptionnelles du sodium foudu comme lluide de transfert de chaleur à haute température et sous faible pression.

La température d'ébullition du sodium à la pression normale étant voisine de $800^{\circ} \mathrm{C}$ à la pression atmosphérique, on peut notamment réaliser un four, chauffé par une circulation de sodium, présentant la majeure partic des avantages des fours électriques à résistance à atmosphère contrôlée, tout en étant alimenté plus économiquement au fuel ou même au charbon.

Dans un domaine tout different, on peut facilement vérifier qu'une pompe type Faraday à très faible entrefer, fonctionnant sous des champs et des courants intenses, peut fournir des pressions de plusieurs dizaines d'atmosphères, qu'il est facile de moduler à volonté.

Ainsi, conclut $M$. le Président, « les diverses pompes électro-magnétiques que j'ai présentées ici même en 1948 (1) et 1949 comme de simples curiosités seront-elles peut-être demain des engins industriels courants $\gg$.

(1) Voir G. Rementeras, «Sur la possibilité de transformer directement en énergie électrique une partie de l'énergie d'une veine fluide » (Mémoires et Travaux de la S.H.F., $\left.\mathrm{n}^{\circ} \mathrm{I}-1.948\right)$ et "Premières Journées de l'Hy'draulique $\gg(1949)$.

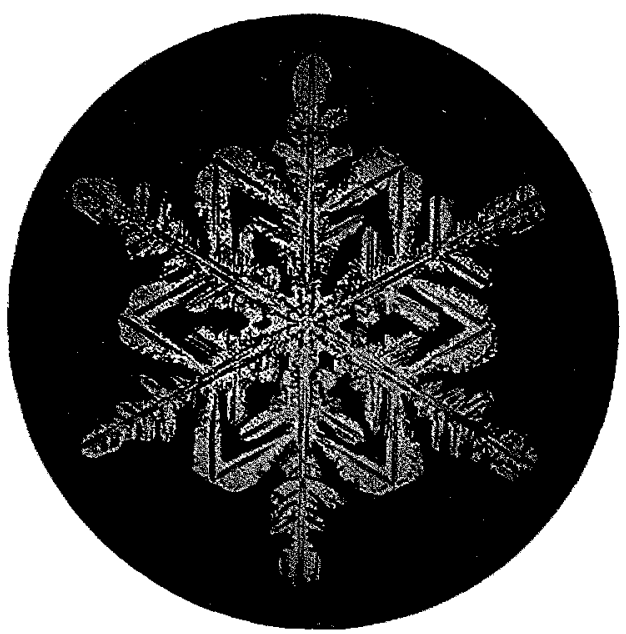

